TRANSACTIONS OF THE

AMERICAN MATHEMATICAL SOCIETY

Volume 356, Number 6, Pages 2443-2457

S 0002-9947(03)03387-7

Article electronically published on November 25, 2003

\title{
DIALGEBRA COHOMOLOGY AS A G-ALGEBRA
}

\author{
ANITA MAJUMDAR AND GOUTAM MUKHERJEE
}

\begin{abstract}
It is well known that the Hochschild cohomology $H^{*}(A, A)$ of an associative algebra $A$ admits a G-algebra structure. In this paper we show that the dialgebra cohomology $H Y^{*}(D, D)$ of an associative dialgebra $D$ has a similar structure, which is induced from a homotopy G-algebra structure on the dialgebra cochain complex $C Y^{*}(D, D)$.
\end{abstract}

\section{INTRODUCTION}

It is well known, since the pioneering work of M. Gerstenhaber [2], that the Hochschild cochain complex $C^{*}(A, A)$ of an associative algebra $A$ admits a brace algebra structure. Moreover, in [3], M. Gerstenhaber and A. A. Voronov have shown that $C^{*}(A, A)$ admits a homotopy $\mathrm{G}$-algebra structure which induces the G-algebra structure on the Hochschild cohomology as introduced in [2]. These structures on $C^{*}(A, A)$ are in fact induced from a natural operad structure on $C^{*}(A, A)$, where only the non- $\Sigma$ part of the operad is responsible for inducing the above structures.

The notions of Leibniz algebras and associative dialgebras were introduced in [6], by J.-L. Loday. Leibniz algebras are a non-commutative variation of Lie algebras, and associative dialgebras are a variation of associative algebras. Recall that an associative algebra gives rise to a Lie algebra by $[x, y]=x y-y x$. The notion of associative dialgebra is introduced in order to build an analogue of the couple

$$
\text { Lie algebras } \leftrightarrow \text { associative algebras, }
$$

when Lie algebras are replaced by Leibniz algebras. A cohomology theory associated with dialgebras has been developed by Loday, called dialgebra cohomology, where in the construction of the dialgebra complex which defines the dialgebra cohomology, planar binary trees play a crucial role. Dialgebra cohomology with coefficients has been studied by A. Frabetti in [1]. In [7, it has been shown that the dialgebra complex $C Y^{*}(D, D)$ admits the structure of an associative algebra, and also of a pre-Lie algebra. The aim of this paper is to show that, as in the case of a Hochschild complex, $C Y^{*}(D, D)$ admits a homotopy G-algebra structure which comes from a non- $\Sigma$ operad structure on $C Y^{*}(D, D)$. As a consequence, the dialgebra cohomology $H Y^{*}(D, D)$ becomes a G-algebra.

Received by the editors September 27, 2002 and, in revised form, April 24, 2003.

2000 Mathematics Subject Classification. Primary 13D03, 17B63; Secondary 18G60.

Key words and phrases. Dialgebra, operad, brace algebra, homotopy G-algebra, G-algebra.

The first author was supported by an N.B.H.M. Research Fellowship. 


\section{Dialgebra COMPlex}

In this section, we recall the construction of a dialgebra complex. Throughout this paper, by dialgebra we mean associative dialgebra.

Definition 2.1. Let $K$ be a field. A dialgebra $D$ over $K$ is a vector space over $K$ along with two $K$-linear maps, $\dashv: D \otimes D \longrightarrow D$ (called left) and $\vdash: D \otimes D \longrightarrow D$ (called right), satisfying the following axioms:

$$
\left\{\begin{array}{l}
x \dashv(y \dashv z) \stackrel{1}{=}(x \dashv y) \dashv z \stackrel{2}{=} x \dashv(y \vdash z), \\
(x \vdash y) \dashv z \stackrel{3}{=} x \vdash(y \dashv z), \\
(x \dashv y) \vdash z \stackrel{4}{=} x \vdash(y \vdash z) \stackrel{5}{=}(x \vdash y) \vdash z,
\end{array}\right.
$$

for all $x, y, z \in D$.

A planar binary tree with $n$ vertices (in short, an $n$-tree) is a planar tree with $(n+1)$ leaves, one root and each vertex trivalent. Let $Y_{n}$ denote the set of all $n$-trees. Let $Y_{0}$ be the singleton set consisting of a root only. The $n$-trees for $0 \leq n \leq 3$ are given by the following diagrams:

$$
\mathrm{Y}_{0}=\{\mid\}, \quad \mathrm{Y}_{1}=\{Y\}, \quad \mathrm{Y}_{2}=\{Y, Y\}, \quad \mathrm{Y}_{3}=\{Y, Y, Y, Y, Y,\}
$$

For any $y \in Y_{n}$, the $(n+1)$ leaves are labelled by $\{0,1, \ldots, n\}$ from left to right, and the vertices are labelled $\{1,2, \ldots, n\}$, so that the $i$ th vertex is between the leaves $(i-1)$ and $i$. Recall from [6] that the only element $\mid$ of $Y_{0}$ is denoted by [0]. The only element of $Y_{1}$ is denoted by [1]. The grafting of a $p$-tree $y_{1}$ and a $q$-tree $y_{2}$ is a $(p+q+1)$-tree denoted by $y_{1} \vee y_{2}$ which is obtained by joining the roots of $y_{1}$ and $y_{2}$ and creating a new root from that vertex. This is denoted by $\left[y_{1} p+q+1 y_{2}\right]$ with the convention that all zeros are deleted except for the element in $Y_{0}$. With this notation, the trees pictured above from left to right are [0], [1], [12], [21], [123], [213], [131], [312], [321].

For any $i, 0 \leq i \leq n$, there is a map, called the face map, $d_{i}: Y_{n} \longrightarrow Y_{n-1}$, $y \mapsto d_{i} y$, where $d_{i} y$ is obtained from $y$ by deleting the $i$ th leaf. The face maps satisfy the relation $d_{i} d_{j}=d_{j-1} d_{i}$, for all $i<j$.

Let $D$ be a dialgebra over a field $K$. The cochain complex $C Y^{*}(D, D)$ which defines the dialgebra cohomology $H Y^{*}(D, D)$ is defined as follows. For any $n \geq 0$, let $K\left[Y_{n}\right]$ denote the $K$-vector space spanned by $Y_{n}$, and let

$$
C Y^{n}(D, D):=\operatorname{Hom}_{K}\left(K\left[Y_{n}\right] \otimes D^{\otimes n}, D\right)
$$

be the module of $n$-cochains of $D$ with coefficients in $D$. The coboundary operator $\delta: C Y^{n}(D, D) \longrightarrow C Y^{n+1}(D, D)$ is defined as the $K$-linear map $\delta=\sum_{i=0}^{n+1}(-1)^{i} \delta^{i}$, where

$$
\left(\delta^{i} f\right)\left(y ; a_{1}, a_{2}, \ldots, a_{n+1}\right):= \begin{cases}a_{1} \circ_{0}^{y} f\left(d_{0} y ; a_{2}, \ldots, a_{n+1}\right), & \text { if } i=0 \\ f\left(d_{i} y ; a_{1}, \ldots, a_{i} \circ{ }_{i}^{y} a_{i+1}, \ldots, a_{n+1}\right), & \text { if } 1 \leq i \leq n \\ f\left(d_{n+1} y ; a_{1}, \ldots, a_{n}\right) \circ_{n+1}^{y} a_{n+1}, & \text { if } i=n+1\end{cases}
$$


for any $y \in Y_{n+1} ; a_{1}, \ldots, a_{n+1} \in D$ and $f: K\left[Y_{n}\right] \otimes D^{\otimes n} \longrightarrow D$. Here, for any $i$, $0 \leq i \leq n+1$, the maps $\circ_{i}: Y_{n+1} \longrightarrow\{\dashv, \vdash\}$ are defined by

$$
\begin{gathered}
\circ_{0}(y)=\circ_{0}^{y}:= \begin{cases}\dashv & \text { if } y \text { is of the form } \mid \vee y_{1}, \text { for some } n \text {-tree } y_{1}, \\
\vdash & \text { otherwise, }\end{cases} \\
\circ_{i}(y)=\circ_{i}^{y}:= \begin{cases}\dashv & \text { if the } i^{\text {th }} \text { leaf of } y \text { is oriented like ' }, \\
\vdash & \text { if the } i^{\text {th }} \text { leaf of } y \text { is oriented like ' } /,\end{cases}
\end{gathered}
$$

for $1 \leq i \leq n$, and

$$
\circ_{n+1}(y)=\circ_{n+1}^{y}:= \begin{cases}\vdash & \text { if } y \text { is of the form } y_{1} \vee \mid, \text { for some } n \text {-tree } y_{1}, \\ \dashv & \text { otherwise, }\end{cases}
$$

where the symbol ' $V$ ' stands for grafting of trees [6].

\section{BRACES For A DiAlgebra COMPLEX}

In this section, we introduce braces or multilinear operations in $C Y^{*}(D, D)$ of a dialgebra $D$, generalizing the $\circ_{i}$ products as introduced in [7], which endow $C Y^{*}(D, D)$ with a brace algebra structure.

Definition 3.1. A brace algebra is a graded vector space with a collection of braces (or multilinear operations) $x\left\{x_{1}, x_{2}, \ldots, x_{n}\right\}$ of degree $-n$ satisfying the identity (brace identity)

$$
x\left\{x_{1}, x_{2}, \ldots, x_{m}\right\}\left\{y_{1}, y_{2}, \ldots, y_{n}\right\}=\sum_{0 \leq i_{1} \leq j_{1} \leq i_{2} \leq \ldots \leq i_{m} \leq j_{m} \leq n}(-1)^{\epsilon} x\left\{y_{1}, \ldots, y_{i_{1}},\right.
$$

where $x\{\}$ is understood as just $x, \operatorname{deg} x\left\{x_{1}, \ldots, x_{n}\right\}=\operatorname{deg} x+\sum_{i=1}^{n} \operatorname{deg} x_{i}-$ $n,|x|=\operatorname{deg} x-1$, and $\epsilon=\sum_{p=1}^{m}\left|x_{p}\right| \sum_{q=1}^{i_{p}}\left|y_{q}\right|$.

Definition 3.2. Let $n, i_{1}, i_{2}, \ldots, i_{r}, m_{1}, m_{2}, \ldots, m_{r}$ be non-negative integers with $n, m_{1}, \ldots, m_{r} \geq 1$ such that

$$
0 \leq i_{1}, i_{1}+m_{1} \leq i_{2}, \ldots, i_{r-1}+m_{r-1} \leq i_{r}, i_{r}+m_{r} \leq N=n+\sum_{1}^{r} m_{i}-r .
$$

For each $j, 0 \leq j \leq r$, we define maps

$$
R_{j+1}^{i_{1}, \ldots, i_{r}}\left(N ; n, m_{1}, \ldots, m_{r}\right): Y_{N} \longrightarrow Y_{m_{j}},
$$

with $m_{0}=n$, in the following way. For $j=0$,

$$
R_{1}^{i_{1}, \ldots, i_{r}}\left(N ; n, m_{1}, \ldots, m_{r}\right)=\prod_{\substack{m_{\ell} \geq 2 \\ 1 \leq \ell \leq r}}\left(d_{i_{\ell}+1} \cdots d_{i_{\ell}+m_{\ell}-1}\right) \text { if } 2 \leq m_{0}<N
$$

where $\Pi$ stands for composition of terms and $R_{1}^{i_{1}, \ldots, i_{r}}\left(N ; n, m_{1}, \ldots, m_{r}\right)$ is the identity or the obvious constant map according to whether $m_{0}$ is $N$ or 1 . 
For $1 \leq j \leq r$, if $2 \leq m_{j}<N$ we have

$$
R_{j+1}^{i_{1}, \ldots, i_{r}}\left(N ; n, m_{1}, \ldots, m_{r}\right)= \begin{cases}\left(d_{0} \cdots d_{i_{j}-1}\right)\left(d_{i_{j}+m_{j}+1} \cdots d_{N}\right), & i_{j} \geq 1 \text { and } \\ & i_{j}+m_{j}+1 \leq N \\ \left(d_{m_{j}+1} \cdots d_{N}\right), & i_{j}=0, \\ \left(d_{0} \cdots d_{i_{j}-1}\right), & i_{j}+m_{j}+1>N\end{cases}
$$

and $R_{j+1}^{i_{1}, \ldots, i_{r}}\left(N ; n, m_{1}, \ldots, m_{r}\right)$ is the identity or the obvious constant map according to whether $m_{j}=N$ or $m_{j}=1$.

Definition 3.3. Let $D$ be a dialgebra over a field $K$. For non-negative integers $n, i_{1}, \ldots i_{r}, m_{1}, \ldots, m_{r}$ with $0 \leq i_{1}, i_{1}+m_{1} \leq i_{2}, \ldots, i_{r-1}+m_{r-1} \leq i_{r}, i_{r}+m_{r} \leq$ $N=n+\sum_{1}^{r} m_{i}-r$, the multilinear maps

$$
\circ_{i_{1}, \ldots, i_{r}}: C Y^{n}(D, D) \otimes \bigotimes_{j=1}^{r} C Y^{m_{j}}(D, D) \longrightarrow C Y^{N}(D, D)
$$

are defined as follows. Let $f \in C Y^{n}(D, D), g_{j} \in C Y^{m_{j}}(D, D), 1 \leq j \leq r$. For $y \in Y_{N}$ and $x_{1}, \ldots, x_{N} \in D$ we have

$$
\begin{aligned}
& f \circ_{i_{1}, \ldots, i_{r}}\left(g_{1}, \ldots, g_{r}\right)\left(y ; x_{1}, \ldots, x_{N}\right) \\
= & f\left(R_{1}^{i_{1}, \ldots, i_{r}}\left(N ; n, m_{1}, \ldots, m_{r}\right) y ; x_{1}, \ldots, x_{i_{1}},\right. \\
& g_{1}\left(R_{2}^{i_{1}, \ldots, i_{r}}\left(N ; n, m_{1}, \ldots, m_{r}\right) y ; x_{i_{1}+1}, \ldots, x_{i_{1}+m_{1}}\right), \ldots, \\
& \left.g_{r}\left(R_{r+1}^{i_{1}, \ldots, i_{r}}\left(N ; n, m_{1}, \ldots, m_{r}\right) y ; x_{i_{r}+1}, \ldots, x_{i_{r}+m_{r}}\right), \ldots, x_{N}\right) .
\end{aligned}
$$

In the above definition, if $m_{j}=0$ for some $j$, then

$$
g_{j} \in C Y^{0}(D, D) \cong \operatorname{Hom}_{K}(K, D)=D
$$

and the corresponding input is simply $g_{j}$.

Next we use these generalized $\circ_{i}$ products to define braces as follows.

Definition 3.4. For $f \in C Y^{n}(D, D), g_{\nu} \in C Y^{m_{\nu}}(D, D), \nu=1, \ldots, r$,

$$
f\left\{g_{1}, \ldots, g_{r}\right\}=\sum_{i_{1}, \ldots, i_{r}}(-1)^{\eta} f \circ_{i_{1}, \ldots, i_{r}}\left(g_{1}, \ldots, g_{r}\right),
$$

where $\eta=\sum_{\nu=1}^{r}\left|g_{\nu}\right| i_{\nu}$, and $\left|g_{\nu}\right|=\operatorname{deg} g_{\nu}-1=m_{\nu}-1$.

Remark 3.5. It may be noted that by the above definition of braces on $C Y^{*}(D, D)$, $f\{g\}$ coincides with the pre-Lie product $f \circ g$ as introduced in [7].

Henceforth, we shall use the symbol $f \circ g$ in order to denote $f\{g\}$. The following proposition will follow from Lemma 5.1

Proposition 3.6. The braces as defined above make the dialgebra cochain complex $C Y^{*}(D, D)$ into a brace algebra.

\section{Operad structure}

In this section we show that the dialgebra complex $C Y^{*}(D, D)$ of a dialgebra $D$ admits the structure of a non- $\Sigma$ operad.

Definition 4.1. A non- $\Sigma$ operad $\mathcal{C}$ of $K$-vector spaces consists of vector spaces $\mathcal{C}(j), j \geq 0$, together with a unit map $K \longrightarrow \mathcal{C}(1)$ and multilinear maps

$$
\gamma: \mathcal{C}(k) \otimes \mathcal{C}\left(j_{1}\right) \otimes \cdots \otimes \mathcal{C}\left(j_{k}\right) \longrightarrow \mathcal{C}(j)
$$

for $k \geq 1 ; j_{s} \geq 0$ and $j=\sum_{s=1}^{k} j_{s}$. The maps $\gamma$ are required to be associative and unital as in [8]. 
The following maps on trees will be used to define a non- $\Sigma$ operad structure on $C Y^{*}(D, D)$.

Definition 4.2. Given an integer $j$, with $j=\sum_{r=1}^{k} j_{r}, k \geq 1$ and $j_{r} \geq 1$, define maps

$$
\begin{array}{ll}
\Gamma^{0}\left(k ; j_{1}, \ldots, j_{k}\right) & : \quad Y_{j} \longrightarrow Y_{k}, \\
\Gamma^{r}\left(k ; j_{1}, \ldots, j_{k}\right) & : \quad Y_{j} \longrightarrow Y_{j_{r}}, 1 \leq r \leq k,
\end{array}
$$

by

$$
\begin{aligned}
& \Gamma^{0}\left(k ; j_{1}, \ldots, j_{k}\right) \\
& \quad=d_{1} \cdots d_{j_{1}-1} d_{j_{1}+1} \cdots d_{j_{1}+j_{2}-1} d_{j_{1}+j_{2}+1} \cdots d_{\sum_{s=1}^{r} j_{s}-1} d_{\sum_{s=1}^{r} j_{s}+1} \cdots \\
& \quad d_{\sum_{s=1}^{k-1} j_{s}-1} d_{\sum_{s=1}^{k-1} j_{s}+1} \cdots d_{j-1} \\
& \quad=d_{1} \cdots \check{d}_{p_{1}} \cdots \check{d}_{p_{2}} \cdots \check{d}_{p_{r}} \cdots \check{d}_{p_{k-1}} \cdots d_{p_{k}-1} \text { for all } 1 \leq r \leq k-1,
\end{aligned}
$$

and

$$
\begin{aligned}
\Gamma^{r}\left(k ; j_{1}, \ldots, j_{k}\right) & =d_{0} \cdots d_{\sum_{s=1}^{r-1} j_{s}-1} d_{\sum_{s=1}^{r} j_{s}+1} \cdots d_{\sum_{s=1}^{k} j_{s}} \\
& =d_{0} \cdots d_{p_{r-1}-1} d_{p_{r}+1} \cdots d_{j}
\end{aligned}
$$

where $p_{r}=j_{1}+j_{2}+\cdots+j_{r}, 1 \leq r \leq k$, and the symbol $\check{d}_{i}$ appearing in any expression means that the map $d_{i}$ has been omitted.

Remark 4.3. Given integers $j, k \geq 1, j_{r} \geq 1$ with $j=\sum_{r=1}^{k} j_{r}$, we shall often write the map $\Gamma^{r}\left(k ; j_{1}, \ldots, j_{k}\right)$ simply as $\Gamma^{r}$, for all $r=0,1, \ldots, k$. However, to avoid confusion we shall write the maps $\Gamma^{r}$ explicitly, along with the values of $k, j_{1}, \ldots, j_{k}$, whenever necessary.

Theorem 4.4. For a dialgebra $D$ over a field $K$, the dialgebra complex $C Y^{*}(D, D)$ is a non- $\Sigma$ operad of $K$-vector spaces.

To prove the above theorem we need the following lemma.

Lemma 4.5. Let $j_{r} \geq 1,1 \leq r \leq k$ be integers with $j=\sum_{r=1}^{k} j_{r}$. Let $i=\sum_{t=1}^{j} i_{t}$, with integers $i_{t} \geq 1$. Set $p_{s}=j_{1}+j_{2}+\cdots+j_{s}$ and $q_{s}=i_{p_{s-1}+1}+\cdots+i_{p_{s}}$. Then for $1 \leq s \leq j_{r}, 1 \leq r \leq k$ the corresponding maps

$$
\begin{aligned}
& \Gamma^{0}\left(k ; j_{1}, \ldots, j_{k}\right): Y_{j} \longrightarrow Y_{k}, \\
& \Gamma^{0}\left(j ; i_{1}, \ldots, i_{j}\right): Y_{i} \longrightarrow Y_{j}, \\
& \Gamma^{0}\left(k ; q_{1}, \ldots, q_{k}\right): Y_{i} \longrightarrow Y_{k}, \\
& \Gamma^{0}\left(j_{r} ; i_{p_{r-1}+1}, \ldots, i_{p_{r-1}+j_{r}}\right): Y_{q_{r}} \longrightarrow Y_{j_{r}}, \\
& \Gamma^{s}\left(j_{r} ; i_{p_{r-1}+1}, \ldots, i_{p_{r-1}+j_{r}}\right): Y_{q_{r}} \longrightarrow Y_{i_{p_{r-1}+s}}, \\
& \Gamma^{r}\left(k ; j_{1}, \ldots, j_{k}\right): Y_{j} \longrightarrow Y_{j_{r}}, \\
& \Gamma^{p_{r-1}+s}\left(j ; i_{1}, \ldots, i_{j}\right): Y_{i} \longrightarrow Y_{i_{p_{r-1}+s}}, \\
& \Gamma^{r}\left(k ; q_{1}, \ldots, q_{k}\right): Y_{i} \longrightarrow Y_{q_{r}}
\end{aligned}
$$

satisfy

(a) $\Gamma^{0}\left(k ; j_{1}, \ldots, j_{k}\right) \Gamma^{0}\left(j ; i_{1}, \ldots, i_{j}\right)=\Gamma^{0}\left(k ; q_{1}, \ldots, q_{k}\right)$,

(b) $\Gamma^{r}\left(k ; j_{1}, \ldots, j_{k}\right) \Gamma^{0}\left(j ; i_{1}, \ldots, i_{j}\right)=\Gamma^{0}\left(j_{r} ; i_{p_{r-1}+1}, \ldots, i_{p_{r-1}+j_{r}}\right) \Gamma^{r}\left(k ; q_{1}, \ldots, q_{k}\right)$,

(c) $\Gamma^{p_{r-1}+s}\left(j ; i_{1}, \ldots, i_{j}\right)=\Gamma^{s}\left(j_{r} ; i_{p_{r-1}+1}, \ldots, i_{p_{r-1}+j_{r}}\right) \Gamma^{r}\left(k ; q_{1}, \ldots, q_{k}\right)$. 
Proof. The above lemma is a repeated application of the simplicial identity $d_{i} d_{j}=$ $d_{j-1} d_{i}, i<j$. We sketch below the proof of (a); the proofs of the other cases are similar. The operator $\Gamma^{0} \Gamma^{0}$ on the left hand side of (a) is given by two strings of operators as

$$
\begin{aligned}
\Gamma^{0} \Gamma^{0}= & \left(d_{1} \cdots \check{d}_{p_{1}} \cdots \check{d}_{p_{2}} \cdots \check{d}_{p_{k-1}} \cdots d_{p_{k}-1}\right) \\
& \left(d_{1} \cdots \check{d}_{i_{1}} \cdots \check{d}_{i_{1}+i_{2}} \cdots \check{d}_{\sum_{t=1}^{j-1} i_{t}} \cdots d_{i-1}\right) .
\end{aligned}
$$

Now that the operator $d_{1}$ at the extreme left in

$$
d_{1} \cdots \check{d}_{p_{1}} \cdots \check{d}_{p_{2}} \cdots \check{d}_{p_{k-1}} \cdots d_{p_{k}-1}
$$

can be brought to the extreme right by successive application of $d_{i} d_{j}=d_{j-1} d_{i}$, $i<j$, yielding

$$
d_{1} \cdots \check{d}_{p_{1}-1} \cdots \check{d}_{p_{2}-1} \cdots \check{d}_{p_{k-1}-1} \cdots d_{p_{k}-2} d_{1} .
$$

Now, by applying $d_{j-1} d_{i}=d_{i} d_{j}, i<j$, the operator $d_{1}$ at the right of the above string can be pushed into the string

$$
d_{1} \cdots \check{d}_{i_{1}} \cdots \check{d}_{i_{1}+i_{2}} \cdots \check{d}_{\sum_{t=1}^{j-1} i_{t}} \cdots d_{i-1}
$$

to recover the operator $d_{i_{1}}$, thus yielding

$$
\begin{aligned}
& \Gamma^{0} \Gamma^{0}=\left(d_{1} \cdots \check{d}_{p_{1}-1} \cdots \check{d}_{p_{2}-1} \cdots \check{d}_{p_{k-1}-1} \cdots d_{p_{k}-2}\right) \\
&\left(d_{1} \cdots d_{i_{1}} \cdots \check{d}_{i_{1}+i_{2}} \cdots \check{d}_{\sum_{t=1}^{j-1} i_{t}} \cdots d_{i-1}\right) .
\end{aligned}
$$

We repeat the above method, each time starting with the operator $d_{1}$ at the left of the first string to recover an omitted operator in the second string. After $\left(p_{1}-1\right)$ steps, we get

$$
\begin{aligned}
\Gamma^{0} \Gamma^{0}= & \left(d_{2} \cdots d_{p_{2}-p_{1}} d_{p_{2}-\left(p_{1}-2\right)} \cdots d_{p_{r}-p_{1}} d_{p_{r}-\left(p_{1}-2\right)} \cdots\right. \\
& \left.d_{p_{k-1}-p_{1}} d_{p_{k-1}-\left(p_{1}-2\right)} \cdots d_{p_{k}-p_{1}}\right)\left(d_{1} \cdots d_{i_{1}} \cdots d_{i_{1}+i_{2}} \cdots\right. \\
& \left.\check{d}_{q_{1}} \cdots \breve{d}_{\sum_{t=1}^{j-1} i_{t}} \cdots d_{i-1}\right)
\end{aligned}
$$

since $q_{1}=i_{1}+\cdots+i_{p_{1}}$. Again we apply the above method starting with the operators $d_{2}, \ldots, d_{p_{2}-p_{1}}$ at the left end of the first string to replace all the omitted operators between $d_{q_{1}+1}$ and $d_{q_{1}+q_{2}-1}$, of the second string. Proceeding this way, all the operators of the first string can be exhausted to yield

$$
\begin{aligned}
\Gamma^{0} \Gamma^{0}= & d_{1} \cdots d_{q_{1}-1} d_{q_{1}+1} \cdots d_{q_{1}+q_{2}-1} d_{q_{1}+q_{2}+1} \cdots \\
& d_{\sum_{s=1}^{r} q_{s}-1} d_{\sum_{s=1}^{r} q_{s}+1} \cdots d_{\sum_{s=1}^{k-1} q_{s}-1} d_{\sum_{s=1}^{k-1} q_{s}+1} \cdots d_{i-1} .
\end{aligned}
$$

Observe that $\sum_{s=1}^{k} q_{s}=i$.

But this is the operator $\Gamma^{0}$ of the right hand side of the equality (a). This proves part (a).

Proof of the theorem. For each $j \geq 0$, set

$$
\mathcal{C}(j)=C Y^{j}(D, D)=\operatorname{Hom}_{K}\left(K\left[Y_{j}\right] \otimes D^{\otimes j}, D\right) .
$$

Note that

$$
\begin{aligned}
\mathcal{C}(1) & =\operatorname{Hom}_{K}\left(K\left[Y_{1}\right] \otimes D, D\right) \\
& \cong \operatorname{Hom}_{K}(D, D) .
\end{aligned}
$$


Define the unit map $\eta: K \longrightarrow \mathcal{C}(1)$ by $\eta(1)=i d_{D}$. Now, for $k \geq 1, j_{r} \geq 0$ and $j=\sum j_{r}$ we define multilinear maps

$$
\gamma: C Y^{k}(D, D) \otimes \bigotimes_{r=1}^{k} C Y^{j_{r}}(D, D) \longrightarrow C Y^{j}(D, D)
$$

as follows: For $f \in C Y^{k}(D, D), g_{r} \in C Y^{j_{r}}(D, D)$

$$
\begin{aligned}
& \gamma\left(f ; g_{1}, \ldots, g_{k}\right)\left(y ; x_{1} \ldots, x_{j}\right) \\
= & f\left(\Gamma^{0}(y) ; g_{1}\left(\Gamma^{1}(y) ; x_{1}, \ldots, x_{j_{1}}\right), g_{2}\left(\Gamma^{2}(y) ; x_{j_{1}+1}, \ldots, x_{j_{1}+j_{2}}\right), \ldots,\right. \\
& \left.g_{k}\left(\Gamma^{k}(y) ; x_{\sum_{s}^{k-1} j_{s}+1}, \ldots, x_{\sum_{s=1}^{k} j_{s}}\right)\right) \\
= & f\left(\Gamma^{0}(y) ; g_{1}\left(\Gamma^{1}(y) ; x_{1}, \ldots, x_{p_{1}}\right), g_{2}\left(\Gamma^{2}(y) ; x_{p_{1}+1}, \ldots, x_{p_{2}}\right), \ldots,\right. \\
& \left.g_{k}\left(\Gamma^{k}(y) ; x_{p_{k-1}+1}, \ldots, x_{p_{k}}\right)\right),
\end{aligned}
$$

where $\Gamma^{0}=\Gamma^{0}\left(k ; j_{1}, \ldots, j_{k}\right): Y_{j} \longrightarrow Y_{k}$, and $\Gamma^{r}=\Gamma^{r}\left(k ; j_{1}, \ldots, j_{k}\right): Y_{j} \longrightarrow Y_{j_{r}}$ are the maps as defined in Definition 4.2 $x_{1}, \ldots, x_{j} \in D$ and $y \in Y_{j}$.

Note that if $j_{r}=0$ for some $r$, then $g_{r} \in C Y^{0}(D, D) \cong \operatorname{Hom}_{K}(K, D)=D$, and the corresponding input in $f$ is simply $g_{r}$.

To check associativity, let $f \in C Y^{k}(D, D), g_{r} \in C Y^{j_{r}}(D, D), r=1, \ldots, k$, and $h_{t} \in C Y^{i_{t}}(D, D), t=1, \ldots, j=\sum_{r=1}^{k} j_{r}$. As in the above lemma, let $i=$ $\sum_{t=1}^{j} i_{t}, p_{s}=j_{1}+j_{2}+\cdots+j_{s}, q_{s}=i_{p_{s-1}+1}+\cdots+i_{p_{s}}$. Also set $q_{(r, s)}=i_{p_{r-1}+1}+$ $i_{p_{r-1}+2}+\cdots+i_{p_{r-1}+s}, 1 \leq s \leq j_{r}$. Then

$$
\gamma \circ(\gamma \otimes i d)\left(\left(f ; g_{1}, \ldots, g_{k}\right), h_{1}, h_{2}, \ldots, h_{j}\right)=\gamma\left(\gamma\left(f ; g_{1}, \ldots, g_{k}\right) ; h_{1}, \ldots, h_{j}\right) .
$$

On the other hand, shuffle yields

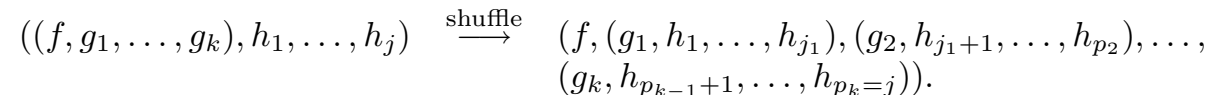

Now, composing with $\gamma \circ\left(i d \otimes\left(\otimes_{r} \gamma\right)\right)$, we get

$$
\begin{aligned}
& \gamma \circ\left(i d \otimes\left(\otimes_{r} \gamma\right)\right) \circ(\text { shuffle })\left(\left(f, g_{1}, \ldots, g_{k}\right), h_{1}, \ldots, h_{j}\right) \\
= & \gamma\left(f ; \gamma\left(g_{1} ; h_{1}, \ldots, h_{p_{1}}\right), \gamma\left(g_{2} ; h_{p_{1}+1}, \ldots, h_{p_{2}}\right), \ldots,\right. \\
& \left.\gamma\left(g_{k} ; h_{p_{k-1}+1}, \ldots, h_{p_{k}=j}\right)\right) .
\end{aligned}
$$

To show that (4.2) and (4.3) are the same cochain in $C Y^{i}(D, D)$, let $y \in Y_{i}$ and $x_{1}, x_{2}, \ldots, x_{i} \in D$. Then,

$$
\begin{aligned}
& \gamma\left(\gamma\left(f ; g_{1}, \ldots, g_{k}\right) ; h_{1}, \ldots, h_{j}\right)\left(y ; x_{1}, \ldots, x_{i}\right) \\
= & \gamma\left(f ; g_{1}, \ldots, g_{k}\right)\left(\Gamma^{0} y ; h_{1}\left(\Gamma^{1} y ; x_{1}, \ldots, x_{i_{1}}\right), h_{2}\left(\Gamma^{2} y ; x_{i_{1}+1}, \ldots, x_{i_{1}+i_{2}}\right),\right. \\
& \left.\ldots, h_{j}\left(\Gamma^{j} y ; x_{\sum_{t=1}^{j-1} i_{t}+1}, \ldots, x_{i}\right)\right),
\end{aligned}
$$

where

$$
\begin{aligned}
\Gamma^{0} y & =\Gamma^{0}\left(j ; i_{1}, \ldots, i_{j}\right) y=d_{1} \cdots \check{d}_{i_{1}} \cdots \check{d}_{i_{1}+i_{2}} \cdots \check{d}_{\sum_{t=1}^{j-1} i_{t}} \cdots d_{i-1} y, \\
\Gamma^{u} y & =\Gamma^{u}\left(j ; i_{1}, \ldots, i_{j}\right) y=d_{0} \cdots d_{\sum_{t=1}^{u-1} i_{t}} d_{\sum_{t=1}^{u} i_{t}+1} \cdots d_{i} y, 1 \leq u \leq j .
\end{aligned}
$$

Now by definition of $\gamma$, as given in (4.1), the equation (4.4) is

$$
\begin{aligned}
= & f\left(\Gamma^{0} \Gamma^{0} y ; g_{1}\left(\Gamma^{1} \Gamma^{0} y ; h_{1}\left(\Gamma^{1} y ; x_{1}, \ldots, x_{i_{1}}\right), \ldots,\right.\right. \\
& \left.h_{j_{1}}\left(\Gamma^{j_{1}} y ; x_{\sum_{t=1}^{j_{1}-1} i_{t}+1}, \ldots, x_{\sum_{t=1}^{j_{1}} i_{t}=q_{1}}\right)\right), \ldots, \\
& g_{k}\left(\Gamma^{k} \Gamma^{0} y ; h_{p_{k-1}+1}\left(\Gamma^{p_{k-1}+1} y ; x_{\sum_{t=1}^{p_{k-1}} i_{t}+1}, \ldots, x_{\sum_{t=1}^{p_{k-1}+1}}{ }_{i_{t}}\right), \ldots,\right. \\
& \left.\left.h_{j}\left(\Gamma^{j} y ; x_{\sum_{t=1}^{j-1} i_{t}+1}^{p_{1}}, \ldots, x_{i}\right)\right)\right)
\end{aligned}
$$


where

$$
\begin{aligned}
\Gamma^{0} \Gamma^{0} y= & \Gamma^{0}\left(k ; j_{1}, \ldots, j_{k}\right) \Gamma^{0}\left(j ; i_{1}, \ldots, i_{j}\right) y \\
= & d_{1} \cdots \check{d}_{p_{1}} \cdots \check{d}_{p_{2}} \cdots \check{d}_{p_{k-1}} \cdots d_{p_{k}-1} d_{1} \\
& \cdots \check{d}_{i_{1}} \cdots \check{d}_{i_{1}+i_{2}} \cdots \check{d}_{\sum_{t=1}^{j-1} i_{t}} \cdots d_{i-1} y
\end{aligned}
$$

and for $1 \leq r \leq k$

$$
\begin{aligned}
\Gamma^{r} \Gamma^{0} y & =\Gamma^{r}\left(k ; j_{1}, \ldots, j_{k}\right) \Gamma^{0}\left(j ; i_{1}, \ldots, i_{j}\right) y \\
& =d_{0} \cdots d_{p_{r-1}-1} d_{p_{r}+1} \cdots d_{p_{k}} d_{1} \cdots \check{d}_{i_{1}} \cdots \check{d}_{i_{1}+i_{2}} \cdots \check{d}_{\sum_{t=1}^{j-1} i_{t}} \cdots d_{i-1} y
\end{aligned}
$$

On the other hand,

$$
\begin{aligned}
& \gamma\left(f ; \gamma\left(g_{1} ; h_{1}, \ldots, h_{p_{1}}\right), \ldots, \gamma\left(g_{k} ; h_{p_{k-1}+1}, \ldots, h_{p_{k}=j}\right)\right)\left(y ; x_{1}, \ldots, x_{i}\right) \\
= & f\left(\Gamma^{0} y ; \gamma\left(g_{1} ; h_{1}, \ldots, h_{p_{1}}\right)\left(\Gamma^{1} y ; x_{1}, \ldots, x_{q_{1}}\right), \ldots,\right. \\
& \left.\gamma\left(g_{k} ; h_{p_{k-1}+1}, \ldots, h_{p_{k}=j}\right)\left(\Gamma^{k} y ; x_{\sum_{s=1}^{k-1} q_{s}+1}, \ldots, x_{\sum_{s=1}^{k} q_{s}=i}\right)\right),
\end{aligned}
$$

where

$$
\begin{aligned}
\Gamma^{0} y & =\Gamma^{0}\left(k ; q_{1}, \ldots, q_{k}\right) y \\
& =d_{1} \cdots \check{d}_{q_{1}} \cdots \check{d}_{q_{1}+q_{2}} \cdots \check{d}_{\sum_{s=1}^{k-1} q_{s}} \cdots d_{\sum_{s=1}^{k} q_{s}-1} y
\end{aligned}
$$

and, for $1 \leq r \leq k$,

$$
\begin{aligned}
\Gamma^{r} y & =\Gamma^{r}\left(k ; q_{1}, \ldots, q_{k}\right) y \\
& =d_{0} \cdots d_{\sum_{s=1}^{r-1} q_{s}-1} d_{\sum_{s=1}^{r} q_{s}+1} \cdots d_{\sum_{s=1}^{k} q_{s}=i} y .
\end{aligned}
$$

By definition of $\gamma$, (4.6) can further be written as

$$
\begin{aligned}
= & f\left(\Gamma^{0} y ; g_{1}\left(\Gamma^{0} \Gamma^{1} y ; h_{1}\left(\Gamma^{1} \Gamma^{1} y ; x_{1}, \ldots, x_{i_{1}}\right), \ldots,\right.\right. \\
& \left.h_{j_{1}}\left(\Gamma^{j_{1}} \Gamma^{1} y ; x_{\sum_{t=1}^{j_{1}-1} i_{t}+1}, \ldots, x_{q_{1}}\right)\right), \ldots, \\
& g_{k}\left(\Gamma^{0} \Gamma^{k} y ; h_{p_{k-1}+1}\left(\Gamma^{1} \Gamma^{k} y ; x_{\sum_{s=1}^{k-1} q_{s}+1}, \ldots, x_{\sum_{t=1}^{p_{k-1}+1}{ }_{i_{t}}}\right), \ldots,\right. \\
& \left.\left.h_{j}\left(\Gamma^{j_{k}} \Gamma^{k} y ; x_{\sum_{t=1}^{j-1} i_{t}+1}, \ldots, x_{i}\right)\right)\right),
\end{aligned}
$$

where

and

$$
\begin{aligned}
\Gamma^{0} \Gamma^{r} y= & \Gamma^{0}\left(j_{r} ; i_{p_{r-1}+1}, \ldots, i_{p_{r-1}+j_{r}}\right) \Gamma^{r}\left(k ; q_{1}, \ldots, q_{k}\right) y \\
= & \left(d_{1} \cdots \dot{d}_{q(r, 1)} \cdots \check{d}_{q(r, 2)} \cdots \dot{d}_{q\left(r, j_{r}-1\right)} \cdots d_{q_{r}-1}\right) \\
& \left(d_{0} \cdots d_{\sum_{s=1}^{r-1} q_{s}-1} d_{\sum_{s=1}^{r} q_{s}+1} \cdots d_{\sum_{s=1}^{k} q_{s}=i}\right) y
\end{aligned}
$$

$$
\begin{aligned}
\Gamma^{s} \Gamma^{r} y= & \Gamma^{s}\left(j_{r} ; i_{p_{r-1}+1}, \ldots, i_{p_{r-1}+j_{r}}\right) \Gamma^{r}\left(k ; q_{1}, \ldots, q_{k}\right) y \\
= & \left(d_{0} \cdots d_{q(r, s-1)-1} d_{q(r, s)+1} \cdots d_{q_{r}}\right) \\
& \left(d_{0} \cdots d_{\sum_{s=1}^{r-1} q_{s}-1} d_{\sum_{s=1}^{r} q_{s}+1}^{r} \cdots d_{\sum_{s=1}^{k} q_{s}=i}\right) y
\end{aligned}
$$

for $1 \leq s \leq j_{r}$ and $1 \leq r \leq k$.

Comparing (4.5) and (4.7), and using Lemma4.5 it follows that the cochains in (4.2) and (4.3) are the same.

To check commutativity of unit diagrams, let $f \in \mathcal{C}(k)=C Y^{k}(D, D)$ and $\alpha_{1}$, $\ldots, \alpha_{k} \in K$. Then,

$$
\gamma \circ\left(\mathrm{id} \otimes \eta^{k}\right)\left(f \otimes\left(\alpha_{1}, \ldots, \alpha_{k}\right)\right)=\gamma\left(f ; \alpha_{1}, \ldots, \alpha_{k}\right),
$$

where we identify $\alpha_{i} \in K$ with the map

$$
\begin{aligned}
\alpha_{i}: \quad & K\left[Y_{1}\right] \otimes D \longrightarrow D, \\
& (y ; a) \mapsto \alpha_{i} a,
\end{aligned}
$$

for all $i=1,2, \ldots, k$. If $\phi$ denotes the isomorphism

$$
\mathcal{C}(k) \otimes K^{k} \cong \mathcal{C}(k)
$$


then

$$
\phi\left(f \otimes\left(\alpha_{1}, \ldots, \alpha_{k}\right)\right)\left(y ; x_{1}, \ldots, x_{k}\right)=f\left(y ; \alpha_{1} x_{1}, \ldots, \alpha_{k} x_{k}\right) .
$$

Now,

$$
\gamma\left(f ; \alpha_{1}, \ldots, \alpha_{k}\right)\left(y ; x_{1}, \ldots, x_{k}\right)=f\left(\Gamma^{0} y ; \alpha_{1}\left(\Gamma^{1} y ; x_{1}\right), \ldots, \alpha_{k}\left(\Gamma^{k} y ; x_{k}\right)\right),
$$

where $\Gamma^{0} y=y$, as $\Gamma^{0}=\Gamma^{0}(k ; 1, \ldots, 1)$ and $\Gamma^{r} y=d_{0} \cdots d_{r-2} d_{r+1} \cdots d_{k} y, 1 \leq r \leq k$.

Therefore,

$$
\gamma\left(f ; \alpha_{1}, \ldots, \alpha_{k}\right)\left(y ; x_{1}, \ldots, x_{k}\right)=f\left(y ; \alpha_{1} x_{1}, \ldots, \alpha_{k} x_{k}\right) .
$$

Hence,

$$
\gamma \circ\left(\mathrm{id} \otimes \eta^{k}\right)\left(f \otimes\left(\alpha_{1}, \ldots, \alpha_{k}\right)\right)=\phi\left(f \otimes\left(\alpha_{1}, \ldots, \alpha_{k}\right)\right) .
$$

Also, for $f \in \mathcal{C}(j)$ and $\alpha \in K$,

$$
\gamma(\eta \otimes \mathrm{id})(\alpha \otimes f)=\gamma(\alpha ; f)
$$

where $\alpha$ is regarded as an element of $\mathcal{C}(1)$ as above.

Now,

$$
\gamma(\alpha ; f)\left(y ; x_{1}, \ldots, x_{j}\right)=\alpha\left(\Gamma^{0} y ; f\left(\Gamma^{1} y ; x_{1}, \ldots, x_{j}\right)\right),
$$

where $\Gamma^{0} y=\Gamma^{0}(1 ; j) y=d_{1} \ldots d_{j-1} y$ and $\Gamma^{1} y=\Gamma^{1}(1 ; j) y=y$. Thus

$$
\begin{aligned}
\gamma(\alpha ; f)\left(y ; x_{1}, \ldots, x_{j}\right) & =\alpha\left(y^{\prime} ; f\left(y ; x_{1}, \ldots, x_{j}\right)\right) \\
& =\alpha f\left(y ; x_{1}, \ldots, x_{j}\right),
\end{aligned}
$$

where $y^{\prime}$ is the only tree in $Y_{1}$.

Note that $\psi: K \otimes \mathcal{C}(j) \stackrel{\cong}{\longrightarrow} \mathcal{C}(j)$ is given by

$$
\psi(\alpha \otimes f)\left(y ; x_{1}, \ldots, x_{j}\right)=\alpha f\left(y ; x_{1}, \ldots, x_{j}\right) .
$$

This completes the proof of the theorem.

\section{BRACES INDUCED BY THE OPERAD STRUCTURE}

We recall from [3] that if $\mathcal{C}(j), j \geq 0$, is a $($ non- $\Sigma)$ operad with multiplication map $\gamma$, then the graded vector space $\mathcal{C}=\bigoplus \mathcal{C}(j)$ admits a brace algebra structure. For $\mathcal{C}(j)=C Y^{j}(D, D)$, the brace algebra structure is given by

$$
f\left\{g_{1}, \ldots, g_{n}\right\}=\sum(-1)^{\epsilon} \gamma\left(f ; \operatorname{id}_{D}, \ldots, \operatorname{id}_{D}, g_{1}, \operatorname{id}_{D}, \ldots, \operatorname{id}_{D}, g_{n}, \operatorname{id}_{D}, \ldots, \operatorname{id}_{D}\right)
$$

where the summation runs over all possible substitutions of $g_{1}, \ldots, g_{n}$ into $f$ in the prescribed order, and $\epsilon=\sum_{p=1}^{n}\left|g_{p}\right| i_{p}, i_{p}$ being the total number of variables one has to input in front of $g_{p}$. Here $\mathrm{id}_{D}$ represents $\eta(1)$. The brace identity is a consequence of the commutativity of associative and unit diagrams. Therefore, in view of Theorem 4.4 we see that $C Y^{*}(D, D)$ admits a brace algebra structure. The following lemma now shows that the braces as introduced in Definition 3.4 make the dialgebra cochain complex into a brace algebra.

Lemma 5.1. The braces on $C Y^{*}(D, D)$ induced by the operad structure coincide with the braces as introduced in Definition 3.4 .

Proof. Let $f \in \mathcal{C}(k)=C Y^{k}(D, D)$ and $g_{i} \in \mathcal{C}\left(m_{i}\right)=C Y^{m_{i}}(D, D), 1 \leq i \leq n$.

Then, according to M. Gerstenhaber and A. A. Voronov [3], the brace induced by the multilinear maps $\gamma$ is given by

$$
f\left\{g_{1}, \ldots, g_{n}\right\}=\sum(-1)^{\epsilon} \gamma\left(f ; \mathrm{id}, \ldots, \mathrm{id}, g_{1}, \mathrm{id}, \ldots, \mathrm{id}, g_{n}, \mathrm{id}, \ldots, \mathrm{id}\right)
$$


where id $=\operatorname{id}_{D}=\eta(1)$ and the summation is over all possible substitutions of $g_{1}, \ldots, g_{n}$ into $f$, in the given order, and $\epsilon=\sum_{p=1}^{n}\left|g_{p}\right| i_{p}, i_{p}$ being the total number of inputs in front of $g_{p}$.

Observe that in the term

$$
(-1)^{\epsilon} \gamma\left(f ; \text { id }, \ldots, \text { id }, g_{1}, \text { id }, \ldots, \text { id }, g_{n}, \text { id }, \ldots, \text { id }\right)
$$

of the above summation, the total number of identity entries in $\gamma$ is $k-n$, the total number of identity entries in front of $g_{1}$ is $i_{1}$ and the total number of identity entries in front of $g_{r}$ is $i_{r}-\sum_{t=1}^{r-1} m_{t}, 2 \leq r \leq n$. Moreover, the following inequalities hold:

$$
0 \leq i_{1}, i_{1}+m_{1} \leq i_{2}, \ldots, i_{r-1}+m_{r-1} \leq i_{r}, i_{n}+m_{n} \leq k+\sum_{t=1}^{n} m_{t}-n=N \text { (say). }
$$

By definition of $\gamma$ as given in (4.1), we have, for $y \in Y_{N}$,

$$
\begin{aligned}
& \gamma\left(f ; \mathrm{id}, \ldots, \mathrm{id}, g_{1}, \mathrm{id}, \ldots, \mathrm{id}, g_{n}, \mathrm{id}, \ldots, \mathrm{id}\right)\left(y ; x_{1}, \ldots, x_{N}\right) \\
= & f\left(\Gamma^{0} y ; x_{1}, \ldots, x_{i_{1}}, g_{1}\left(\Gamma^{i_{1}+1} y ; x_{i_{1}+1}, \ldots, x_{i_{1}+m_{1}}\right), x_{i_{1}+m_{1}+1}, \ldots, x_{i_{2}},\right. \\
& g_{2}\left(\Gamma^{i_{2}-m_{1}+2} y ; x_{i_{2}+1}, \ldots, x_{i_{2}+m_{2}}\right), x_{i_{2}+m_{2}+1}, \ldots, x_{i_{n}}, \\
& \left.g_{n}\left(\Gamma^{i_{n}-\sum_{t=1}^{n-1} m_{t}+n} y ; x_{i_{n}+1}, \ldots, x_{i_{n}+m_{n}}\right), \ldots, x_{N}\right),
\end{aligned}
$$

where

$$
\begin{aligned}
\Gamma^{p}= & \Gamma^{p}(k ; \underbrace{1, \ldots, 1}_{i_{1}}, m_{1}, \underbrace{1, \ldots, 1}_{i_{2}-m_{1}-i_{1}}, m_{2}, \ldots, m_{r-1}, \underbrace{1, \ldots, 1}_{i_{r}-m_{r-1}-i_{r-1}}, \\
& m_{r}, 1, \ldots, m_{n}, \underbrace{1, \ldots, 1}_{N-m_{n}-i_{n}})
\end{aligned}
$$

for $0 \leq p \leq k$. Note that in the definition of $\gamma$ as given in (4.1), the map $\Gamma^{r}$ yields the only tree in $Y_{1}$ when operated on $y$ if $j_{r}=1$ by Definition 4.2. In other words, $\Gamma^{r}$ is the obvious constant map. For instance, by Definition 4.2, the map $\Gamma^{i_{1}+2}$ appearing in (5.1) is given by

$$
\begin{aligned}
\Gamma^{i_{1}+2} & =d_{0} \cdots d_{\left(i_{1}+m_{1}+1\right)-1} d_{\left(i_{1}+m_{1}+2\right)+1} \cdots d_{N} \\
& =d_{0} \cdots d_{i_{1}+m_{1}+1} d_{i_{1}+m_{1}+2} \cdots d_{N}
\end{aligned}
$$

and consists of $N-1$ face maps $d_{i}$; hence $\Gamma^{i_{1}+2} y=y^{\prime}$, where $y^{\prime}$ is the only tree in $Y_{1}$. Hence the corresponding input id $\left(y^{\prime} ; x_{i}\right)$ in $\gamma$ is simply $x_{i}$.

Now according to Definition 4.2 we have

$$
\begin{aligned}
\Gamma^{0}= & \check{d}_{1} \cdots \check{d}_{i_{1}} d_{i_{1}+1} \cdots d_{i_{1}+m_{1}-1} \check{d}_{i_{1}+m_{1}} \cdots \check{d}_{i_{2}} d_{i_{2}+1} \cdots d_{i_{2}+m_{2}-1} \check{d}_{i_{2}+m_{2}} \\
& \cdots \check{d}_{i_{3}} \cdots \check{d}_{i_{r}+m_{r}} \cdots \check{d}_{i_{r+1}} \cdots \check{d}_{i_{n}+m_{n}} \cdots \breve{d}_{N} \\
= & d_{i_{1}+1} \cdots d_{i_{1}+m_{1}-1} d_{i_{2}+1} \cdots d_{i_{2}+m_{2}-1} \cdots d_{i_{r}+1} \\
& \cdots d_{i_{r}+m_{r}-1} \cdots d_{i_{n}+1} \cdots d_{i_{n}+m_{n}-1} \\
= & R_{1}^{i_{1}, \ldots, i_{n}}, \text { as introduced in Definition 3.2. }
\end{aligned}
$$

Also the operator $\Gamma^{i_{r}-\sum_{t=1}^{r-1} m_{t}+r}$, corresponding to $g_{r}$, is given by

$$
\begin{aligned}
& \Gamma^{i_{r}-\sum_{t=1}^{r-1} m_{t}+r} \\
& \quad=d_{0} \cdots d_{\left(i_{r}-\sum_{t=1}^{r-1} m_{t}\right)+\sum_{t=1}^{r-1} m_{t}-1} d_{\left(i_{r}-\sum_{t=1}^{r-1} m_{t}\right)+\sum_{t=1}^{r-1} m_{t}+m_{r}+1} \cdots d_{N}
\end{aligned}
$$

Recall that the number of identity entries in front of $g_{r}$ is $i_{r}-\sum_{t=1}^{r-1} m_{t}$ and their degrees sum up to $i_{r}-\sum_{t=1}^{r-1} m_{t}$, while the sum of the degrees of $g_{1}, \ldots, g_{r-1}$ 
is $\sum_{t=1}^{r-1} m_{t}$. Thus,

$$
\begin{aligned}
\Gamma^{i_{r}-\sum_{t=1}^{r-1} m_{t}+r} & =d_{0} \cdots d_{i_{r}-1} d_{i_{r}+m_{r}+1} \cdots d_{N} \\
& =R_{r+1}^{i_{1}, \ldots, i_{n}}, \text { as introduced in Definition 3.2. }
\end{aligned}
$$

It follows that the $N$-cochain

$$
\gamma\left(f ; \text { id }, \ldots, \text { id }, g_{1}, \text { id }, \ldots, \text { id }, g_{n}, \text { id }, \ldots, \text { id }\right)
$$

is the same as $f \circ_{i_{1}, \ldots, i_{n}}\left(g_{1}, \ldots, g_{n}\right)$. This sets up a sign-preserving bijective correspondence between the terms of the summation

$$
\sum(-1)^{\epsilon} \gamma\left(f ; \text { id }, \ldots, \text { id }, g_{1}, \text { id }, \ldots, \text { id }, g_{n}, \text { id }, \ldots, \text { id }\right)
$$

where the summation is over all possible substitutions of $g_{1}, \ldots, g_{n}$ into $f$, in the given order, $\epsilon=\sum_{p=1}^{n}\left|g_{p}\right| i_{p}, i_{p}$ being the total number of inputs in front of $g_{p}$, and the terms of the summation

$$
\sum(-1)^{\eta} f \circ_{i_{1}, \ldots i_{n}}\left(g_{1}, \ldots, g_{n}\right)
$$

where the summation is over all $i_{1}, \ldots, i_{n}$ such that $0 \leq i_{1}, i_{1}+m_{1} \leq i_{2}, \ldots, i_{n-1}+$ $m_{n-1} \leq i_{n}, i_{n}+m_{n} \leq k+\sum_{i=1}^{n} m_{i}-n$ and $\eta=\sum_{p=1}^{n}\left|g_{p}\right| i_{p}$.

Thus the braces as defined in section 3 are precisely the braces induced by the (non- $\Sigma$ ) operad structure.

\section{G-Algebra structure on COHOMOlOGy}

In this final section we show that the dialgebra cohomology $H Y^{*}(D, D)$ of a dialgebra $D$ has a G-algebra structure which is induced from a homotopy G-algebra structure on the dialgebra cochain complex $C Y^{*}(D, D)$ with the differential altered by a sign.

Let us first recall the following definitions from [3].

Definition 6.1. A homotopy G-algebra is a brace algebra $V=\bigoplus_{n} V^{n}$ provided with a differential $d$ of degree one and a dot product $x \cdot y$ of degree zero making $V$ into a differentially graded associative algebra. The dot product must satisfy the following compatibility identities:

$$
\left(x_{1} \cdot x_{2}\right)\left\{y_{1}, \ldots, y_{n}\right\}=\sum_{k=0}^{n}(-1)^{\epsilon} x_{1}\left\{y_{1}, \ldots, y_{k}\right\} \cdot x_{2}\left\{y_{k+1}, \ldots, y_{n}\right\}
$$

where $\epsilon=\left(\left|x_{2}\right|+1\right) \sum_{p=1}^{k}\left|y_{p}\right|$, and

$$
\begin{aligned}
& d\left(x\left\{x_{1}, \ldots, x_{n+1}\right\}\right)-(d x)\left\{x_{1}, \ldots, x_{n+1}\right\} \\
& -(-1)^{|x|} \sum_{i=1}^{n+1}(-1)^{\left|x_{1}\right|+\cdots+\left|x_{i-1}\right|} x\left\{x_{1}, \ldots, d x_{i}, \ldots x_{n+1}\right\} \\
= & (-1)^{(|x|+1)\left|x_{1}\right|} x_{1} \cdot x\left\{x_{2}, \ldots, x_{n+1}\right\} \\
& -(-1)^{|x|} \sum_{i=1}^{n}(-1)^{\left|x_{1}\right|+\cdots+\left|x_{i}\right|} x\left\{x_{1}, \ldots, x_{i} \cdot x_{i+1}, \ldots x_{n+1}\right\} \\
& +(-1)^{|x|+\left|x_{1}\right|+\cdots\left|x_{n}\right|} x\left\{x_{1}, \ldots, x_{n}\right\} \cdot x_{n+1} .
\end{aligned}
$$

Remark 6.2. It should be mentioned here that the notion of homotopy G-algebras as defined above is different from the notion of strong homotopy G-algebras $\left(\mathcal{G}_{\infty^{-}}\right.$ algebras, for short) as considered in [4]. A $\mathcal{G}_{\infty}$-algebra is an algebra over the minimal model of the Koszul operad describing G-algebras. However, the notion of homotopy G-algebras that we are considering do not really fit the general scheme of quadratic operad theory [5]. 
Definition 6.3. A multiplication on an operad $\mathcal{C}$ of vector spaces is an element $m \in \mathcal{C}(2)$ such that $m \circ m=0$, where $m \circ m:=m\{m\}$ and \{\} denote the associated braces.

The following lemma shows that the operad $C Y^{*}(D, D)$ is equipped with a multiplication.

Lemma 6.4. The 2-cochain $\pi \in C Y^{2}(D, D)$ defined by

$$
\left\{\begin{array}{l}
\pi([21] ; a, b)=a \dashv b \\
\pi([12] ; a, b)=a \vdash b
\end{array}\right.
$$

for all $a, b \in D$ is a multiplication on the operad $C Y^{*}(D, D)$.

Proof. By Remark 3.5, we only need to verify that $\pi \circ \pi=0$. Now, by definition of pre-Lie product as introduced in [7], we have, for $y \in Y_{3}$ and $a, b, c \in D$,

$$
\pi \circ \pi(y ; a, b, c)=\left(\pi \circ_{0} \pi-\pi \circ_{1} \pi\right)(y ; a, b, c) .
$$

The proof now follows from the dialgebra axioms.

In order to show that the dialgebra cochain complex $C Y^{*}(D, D)$ admits a homotopy G-algebra structure, we shall make use of Proposition 2(3) from [3], which we describe below. Let $\mathcal{C}$ denote an operad, $m$ a multiplication on $\mathcal{C}$, and let $m \circ x$ denote $m\{x\}$.

Proposition 6.5. The product

$$
x \cdot y:=(-1)^{|x|+1} m\{x, y\}
$$

of degree 0 and the differential

$$
d x=m \circ x-(-1)^{|x|} x \circ m, d^{2}=0, \operatorname{deg} d=1,
$$

define the structure of a differential graded $(D G)$ associative algebra on $\mathcal{C}$.

First, we observe the following two facts.

Remark 6.6. Note that by Lemma 6.12 of [7], the coboundary operator

$$
\delta: C Y^{n}(D, D) \longrightarrow C Y^{n+1}(D, D)
$$

can be expressed in the form

$$
\delta f=(-1)^{|f|}\left(\pi \circ f-(-1)^{|f|} f \circ \pi\right)=(-1)^{|f|} d f .
$$

Remark 6.7. The $*$ product, as introduced in Definition 6.8 of [7], can be expressed in terms of braces as

$$
f * g=(-1)^{(|f|+1)(|g|)} \pi\{f, g\} .
$$


This is because, by the definition of braces on $C Y^{*}(D, D)$,

$$
\begin{aligned}
\pi\{f, g\}\left(y ; x_{1}, \ldots, x_{p+q}\right)= & (-1)^{p(q-1)} \pi \circ_{0, p}(f, g)\left(y ; x_{1}, \ldots, x_{p+q}\right) \\
= & (-1)^{p(q-1)} \pi\left(R_{1}^{0, p}(p+q ; 2, p, q) y\right. \\
& f\left(R_{2}^{0, p}(p+q ; 2, p, q) y ; x_{1}, \ldots, x_{p}\right), \\
& \left.g\left(R_{3}^{0, p}(p+q ; 2, p, q) y ; x_{p+1}, \ldots, x_{p+q}\right)\right) \\
= & (-1)^{p(q-1)} \pi\left(d_{1} \cdots d_{p-1} d_{p+1} \cdots d_{p+q-1}(y) ;\right. \\
& f\left(d_{p+1} \cdots d_{p+q}(y) ; x_{1}, \ldots x_{p}\right) \\
& \left.g\left(d_{0} \cdots d_{p-1}(y) ; x_{p+1}, \ldots, x_{p+q}\right)\right) \\
= & (-1)^{p(q-1)} \pi\left(d_{1} \cdots d_{p-1} d_{p+1} \cdots d_{p+q-1}(y) ;\right. \\
& f\left(d_{p+1} d_{p+1} \cdots d_{p+q-1}(y) ; x_{1}, \ldots x_{p}\right) \\
& \left.g\left(d_{0} \cdots d_{p-1}(y) ; x_{p+1}, \ldots, x_{p+q}\right)\right) \\
= & (-1)^{p(q-1)} \pi\left(R_{1}^{0}(p+1 ; 2, p) R_{1}^{p}(p+q ; p+1, q)(y) ;\right. \\
& f\left(R_{2}^{0}(p+1 ; 2, p) R_{1}^{p}(p+q ; p+1, q)(y) ; x_{1}, \ldots, x_{p}\right), \\
& g\left(R_{2}^{p}(p+q ; p+1, q)\left((y) ; x_{p+1}, \ldots, x_{p+q}\right)\right) \\
= & (-1)^{p(q-1)} f * g\left(y ; x_{1}, \ldots, x_{p+q}\right) .
\end{aligned}
$$

Here we make use of the fact that the operator $d_{p+q}$ in the string of operators $d_{p+1} \cdots d_{p+q}$ can be moved to the extreme left of the same string using $d_{i} d_{j}=$ $d_{j-1} d_{i}, i<j$, to yield $d_{p+1} d_{p+1} \cdots d_{p+q-1}$.

Therefore by equation (6.5), the dot product $f \cdot g$ determined by the multiplication $m$ as in Proposition 6.5 is in this case nothing but the $*$ product, up to the $\operatorname{sign}(-1)^{(|f|+1)(|g|+1)}$. Moreover, the differential $d$ determined by $m$ as in Proposition 6.5 is merely the coboundary operator $\delta$, up to the sign $(-1)^{(|f|)}$; that is, $d f=(-1)^{(|f|)} \delta(f)$.

Consequently, by Proposition 6.5 and Theorem 4.4 we deduce the following corollary.

Corollary 6.8. The graded cochain module $C Y^{*}(D, D)$ equipped with the $*$ product $f * g$, as introduced in [7, altered by the sign $(-1)^{(|f|+1)(|g|+1)}$ and the coboundary $d f=(-1)^{|f|} \delta f$, is a differential graded associative algebra.

Next we recall Theorem 3 of 3 .

Theorem 6.9. A multiplication on an operad $\mathcal{C}$ defines the structure of a homotopy G-algebra on $\bigoplus_{k} \mathcal{C}(k)$. A multiplication on a brace algebra is equivalent to the structure of a homotopy G-algebra on it.

Thus in view of Theorem 4.4 Theorem[6.9] and Lemma 4.5 we have the following corollary.

Corollary 6.10. The cochain complex $\left(C Y^{*}(D, D), d\right)$, where $d f=(-1)^{|f|} \delta f$, is a homotopy G-algebra with the dot product $f \cdot g=(-1)^{(|f|+1)(|g|+1)} f * g$.

As a consequence, we have the following corollary.

Corollary 6.11. The cochain complex $\left(C Y^{*}(D, D), d\right)$ is a differential graded Lie algebra with respect to the commutator $[x, y]=x \circ y-(-1)^{|x||y|} y \circ x$.

Proof. The brace identity, for $m=n=1$, implies that

$$
x\left\{x_{1}\right\}\left\{y_{1}\right\}=x\left\{x_{1}, y_{1}\right\}+x\left\{x_{1}\left\{y_{1}\right\}\right\}+(-1)^{\left|x_{1}\right|\left|y_{1}\right|} x\left\{y_{1}, x_{1}\right\},
$$

as $0 \leq i_{1} \leq j_{1} \leq 1$. 
Using Remark 3.5, we deduce from above that

$$
\left(x \circ x_{1}\right) \circ y_{1}-x \circ\left(x_{1} \circ y_{1}\right)=x\left\{x_{1}, y_{1}\right\}+(-1)^{\left|x_{1}\right|\left|y_{1}\right|} x\left\{y_{1}, x_{1}\right\} .
$$

A straightforward computation using equation (6.6) and the fact that $|x \circ y|=$ $|x|+|y|$ shows that the commutator satisfies the graded Jacobi identity.

Moreover, the dot product is always homotopy graded commutative; that is,

$$
x \cdot y-(-1)^{(|x|+1)(|y|+1)} y \cdot x=(-1)^{|x|}\left(d(x \circ y)-d x \circ y-(-1)^{|x|} x \circ d y\right) .
$$

This follows directly from equation (6.2), as

$$
\begin{aligned}
& (-1)^{|x|}\left(d(x \circ y)-d x \circ y-(-1)^{|x|} x \circ d y\right) \\
& =(-1)^{|x|}\left((-1)^{(|x|+1)|y|} y \cdot x+(-1)^{|x|} x \cdot y\right) \\
& =x \cdot y-(-1)^{(|x|+1)(|y|+1)} y \cdot x .
\end{aligned}
$$

Also, the differential is a derivation of the bracket. In other words,

$$
d[x, y]-[d x, y]-(-1)^{|x|}[x, d y]=0,
$$

which is a direct consequence of the homotopy graded commutativity of the dot product. This shows that every homotopy G-algebra is a differential graded Lie algebra with respect to the commutator $[x, y]=x \circ y-(-1)^{|x||y|} y \circ x$.

Next we recall the following definition from [3].

Definition 6.12. A G-algebra is a graded vector space $H$ with a dot prduct $x \cdot y$ defining the structure of a graded commutative algebra with a bracket $[x, y]$ of degree -1 defining the structure of a graded Lie algebra such that the bracket with an element is a derivation of the dot product:

$$
[x, y \cdot z]=[x, y] \cdot z+(-1)^{|x|(|y|+1)} y \cdot[x, z] .
$$

Corollary 6.13. The $*$ product $x * y$, altered by the sign $(-1)^{(|x|+1)(|y|+1)}$ and the bracket $[x, y]=x \circ y-(-1)^{|x||y|} y \circ x$, defines the structure of a G-algebra on the dialgebra cohomology $H Y^{*}(D, D)$ of a dialgebra $D$.

Proof. First observe that

$$
H Y^{n}(D, D)=H^{n}\left(\left(C Y^{*}(D, D), \delta\right)\right)=H^{n}\left(\left(C Y^{*}(D, D), d\right)\right)
$$

The fact that the dot product $x \cdot y=(-1)^{|x|+1} \pi\{x, y\}$ lifts to the cohomology follows from Proposition 6.5 Equation (6.7) implies that this dot product is graded commutative. Moreover, by Corollory 6.11, the bracket $[x, y]=x \circ y-(-1)^{|x||y|} y \circ x$ of degree -1 defines the structure of a graded Lie algebra on $H Y^{*}(D, D)$. It remains to show that the bracket with an element is a derivation of the dot product.

First we show that the commutator $[x, y]=x \circ y-(-1)^{|x||y|} y \circ x$ for all $x, y \in$ $C Y^{*}(D, D)$ is a graded derivation of the dot product up to null homotopy; that is,

$$
\begin{aligned}
& {[x, y \cdot z]-[x, y] \cdot z-(-1)^{|x|(|y|+1)} y \cdot[x, z] } \\
= & (-1)^{|x|+|y|+1}\left(d(x\{y, z\})-(d x)\{y, z\}-(-1)^{|x|} x\{d y, z\}-(-1)^{|x|+|y|} x\{y, d z\}\right) .
\end{aligned}
$$


By definition of the commutator, we have

$$
\begin{aligned}
& {[x, y \cdot z]-[x, y] \cdot z-(-1)^{|x|(|y|+1)} y \cdot[x, z] } \\
&= x \circ(y \cdot z)-(-1)^{|x||y \cdot z|}(y \cdot z) \circ x-\left(x \circ y-(-1)^{|x||y|} y \circ x\right) \cdot z \\
&-(-1)^{|x|(|y|+1)} y \cdot\left(x \circ z-(-1)^{|x||z|} z \circ x\right) \\
&=\left(x \circ(y \cdot z)-(-1)^{|x|(|y|+1)} y \cdot(x \circ z)-(x \circ y) \cdot z\right) \\
&-(-1)^{|x||y \cdot z|}(y \cdot z) \circ x-(-1)^{|x||y|}(y \circ x) \cdot z+(-1)^{|x|(|y|+|z|+1)} y \cdot(z \circ x) \\
&=\left(x \circ y \cdot z-(-1)^{|x|(|y|+1)} y \cdot(x \circ z)-(x \circ y) \cdot z\right) \\
&-(-1)^{|x|(|y|+|z|+1)}\left((y \cdot z) \circ x-y \cdot(z \circ x)+(-1)^{|x|(|z|+1)} y \circ x . z\right) \\
&= x \circ y \cdot z-(-1)^{|x|(|y|+1)} y \cdot(x \circ z)-(x \circ y) \cdot z
\end{aligned}
$$

$\left(\right.$ as $\left((y \cdot z) \circ x-y \cdot(z \circ x)+(-1)^{|x|(|z|+1)} y \circ x . z\right)=0$, by equation $\left.(6.1)\right)$

$$
\begin{array}{r}
=(-1)^{|x|+|y|+1}\left(d(x\{y, z\})-(d x)\{y, z\}-(-1)^{|x|} x\{d y, z\}\right. \\
\left.-(-1)^{|x|+|y|} x\{y, d z\}\right)
\end{array}
$$

by equation (6.2). This implies that $[x, y \cdot z]=[x, y] \cdot z+(-1)^{|x|(|y|+1)} y \cdot[x, z]$ for all $x, y, z \in H Y^{*}(D, D)$. Thus $H Y^{*}(D, D)$ admits a G-algebra structure.

\section{REFERENCES}

[1] Frabetti, A. Dialgebra (co)homology with coefficients, Dialgebras and Related Operads, Lecture Notes in Mathematics, Vol 1763, Springer, Berlin, 2001, pp. 67-103. MR 2002i:17003

[2] Gerstenhaber, M. The Cohomology Structure of an Associative Ring, Ann. of Math. 78 (1963) 267-288. MR 28:5102

[3] Gerstenhaber, M. and Voronov, A. A. Homotopy G-algebras and moduli space operad, Internat. Math. Res. Notices 3 (1995), 141-153. MR 96c:18004

[4] Ginot, G. Homology et modèle minimal des algèbres de Gerstenhaber, preprint 2002, http://www-irma.u-strasbg.fr/ ginot/gerstenhaber.ps.

[5] Ginzburg, V. and Kapranov, M. M. Koszul duality for operads, Duke Math Journal, 76(1) (1994) 203-272. MR 96a:18004

[6] Loday, J.-L. Dialgebras, Dialgebras and Related Operads, Lecture Notes in Mathematics, Vol 1763, Springer, Berlin, 2001, pp. 7-66. MR 2002i:17004

[7] Majumdar, A. and Mukherjee, G. Deformation theory of dialgebras, K-theory 27 (2002), 33-60.

[8] May, J. P. Definitions: operads, algebras and modules, Operads: Proceedings of Renaissance Conferences, Contemporary Mathematics, Vol 202, AMS, Providence, RI, 1997, pp. 1-7. MR 97m:18001

Stat-Math Unit, Indian Statistical Institute, 203, B. T. Road, Kolkata-700108, India E-mail address: anita_r@isical.ac.in

Stat-Math Unit, Indian Statistical Institute, 203, B. T. Road, Kolkata-700108, India

E-mail address: goutam@isical.ac.in 